OPEN ACCESS

DUNDEE

\author{
University of Dundee
}

\title{
Quantitative assessment of the mechanical properties of prostate tissue with optical coherence elastography
}

Ling, Yuting; Li, Chunhui; Zhou, Kanheng; Guan, Guangying; Lang, Stephen; McGloin, David; Nabi, Ghulam; Huang, Zhihong

Published in:

Proceedings Volume 10468

DOI:

$10.1117 / 12.2288485$

Publication date:

2018

Document Version

Peer reviewed version

Link to publication in Discovery Research Portal

Citation for published version (APA):

Ling, Y., Li, C., Zhou, K., Guan, G., Lang, S., McGloin, D., ... Huang, Z. (2018). Quantitative assessment of the mechanical properties of prostate tissue with optical coherence elastography. In H. W. Kang, \& K. F. Chan (Eds.), Proceedings Volume 10468: Therapeutics and Diagnostics in Urology 2018 (Vol. 10468). [104680A] (Proceedings of the SPIE; Vol. 10468). Washington: SPIE-International Society for Optical Engineering. https://doi.org/10.1117/12.2288485

\section{General rights}

Copyright and moral rights for the publications made accessible in Discovery Research Portal are retained by the authors and/or other copyright owners and it is a condition of accessing publications that users recognise and abide by the legal requirements associated with these rights.

- Users may download and print one copy of any publication from Discovery Research Portal for the purpose of private study or research. - You may not further distribute the material or use it for any profit-making activity or commercial gain.

- You may freely distribute the URL identifying the publication in the public portal. 


\title{
Quantitative Assessment of the Mechanical Properties of Prostate Tissue with Optical Coherence Elastography
}

\author{
Yuting Ling ${ }^{1,2, \ddagger}$, Chunhui $\mathrm{Li}^{1,2,{ }^{\prime}}$, Kanheng Zhou ${ }^{1,2}$, Guangying Guan ${ }^{1,2}$, Stephen Lang ${ }^{3}$, \\ David McGloin ${ }^{2}$, Ghulam Nabi ${ }^{1}$, Zhihong Huang ${ }^{1,2}$ \\ ${ }^{1}$ Division of Cancer Research, University of Dundee, Ninewells Hospital and Medical School, \\ Dundee DD1 9SY, UK; ${ }^{2}$ School of Science and Engineering, University of Dundee, Dundee, DD1 \\ 4HN, UK; ${ }^{3}$ Department of Pathology, University of Dundee, Ninewells Hospital and Medical \\ School, Dundee DD1 9SY, UK; \#: These authors contributed equally to this work
}

\begin{abstract}
Prostate cancer (PCa) is a heterogeneous disease with multifocal origin. In current clinical care, the Gleason scoring system is the well-established diagnosis by microscopic evaluation of the tissue from trans-rectal ultrasound (TRUS) guided biopsies. Nevertheless, the sensitivity and specificity in detecting PCa can range from 40 to $50 \%$ for conventional TRUS B-mode imaging. Tissue elasticity is associated with the disease progression and elastography technique has recently shown promise in aiding PCa diagnosis. However, many cancer foci in the prostate gland has very small size less than 1 $\mathrm{mm}$ and those detected by medical elastography were larger than $2 \mathrm{~mm}$. Hereby, we introduce optical coherence elastography (OCE) to quantify the prostate stiffness with high resolution in the magnitude of $10 \mu \mathrm{m}$. Following our feasibility study of 10 patients reported previously, we recruited 60 more patients undergoing 12-core TRUS guided biopsies for suspected PCa with a total of 720 biopsies. The stiffness of cancer tissue was approximately $57.63 \%$ higher than that of benign ones. Using histology as reference standard and cut-off threshold of $600 \mathrm{kPa}$, the data analysis showed sensitivity and specificity of $\mathbf{8 9 . 6 \%}$ and $99.8 \%$ respectively. The method also demonstrated potential in characterising different grades of PCa based on the change of tissue morphology and quantitative mechanical properties. In conclusion, quantitative OCE can be a reliable technique to identify PCa lesion and differentiate indolent from aggressive cancer.
\end{abstract}

Keywords: Prostate cancer, Gleason score, elasticity, optical coherence elastography

\section{INTRODUCTION}

Prostate adenocarcinoma (PCa) is the most common cancer in men in the UK. In the routine clinical care, patients are suspected with PCa if they have a raised serum prostate-specific antigen (PSA) level and/or abnormal digital rectal examination (DRE) result. Conventional ultrasound is a popular clinical imaging technique for lesion anatomy based on the acoustic properties of soft tissues, but many soft tissues can share similar ultrasonic echogenicity. In prostate ultrasound imaging, trans-rectal ultrasound (TRUS) guided biopsy is already in use clinically around the world to guide prostate biopsies. However, tumours of prostate may be invisible or barely invisible in standard ultrasound examinations, which leads to unreliable assessment of the tumour volume ${ }^{1}$ and cancer staging ${ }^{2,3}$. The cancer staging is confirmed by the histopathologic verification under microscope using Gleason scoring system ${ }^{4}$ that is relied on the knowledge and experience of the histopathologists. Generally, a higher Gleason score means a more aggressive tumour and a worse prognosis. However, an inter-observer variation exists between two histopathologists besides a large discrepancy existing on initial biopsy and after the final radical prostatectomy (RP). Cohen et al. ${ }^{2}$ conducted a meta-analysis of 14839 patients worldwide, who were diagnosed of PCa with TRUS biopsy and then underwent RP. It was found that only $58 \%$ of the patients in whom the RP grade was accurately predicted. In addition, it is controversial about the sensitivity and specificity of the technique, as well as associated risks of overdiagnosis and overtreatment. Therefore, a quantitative diagnosis is of high demand to make an optimal decision for PCa treatment. 
The differing elasticity of soft tissue has been an established practice for centuries to differentiate healthy from diseased tissue, especially in breast and prostate tissue. PCa was traditionally diagnosed with DRE of the prostate by feeling the stiffness alteration in the prostate. Initially, the mechanical evaluation of prostate elasticity was investigated by Krouskop et al. ${ }^{5}$ with displacement loading experiments. It was found that tissue from PCa has a measurable elevated Young's modulus compared with the normal prostate glandular tissue, and tissue from prostate with benign prostatic hyperplasia $(\mathrm{BPH})$ is significantly softer than normal tissue. Elastography enables deeper tissue elasticity imaging based on the assumption that soft tissue is a linear elastic solid with isotropic mechanical properties ${ }^{6,7}$. Ultrasound elastography has become a commonly used approach for clinical elasticity imaging especially quasi-static ultrasound based elastography ${ }^{8}$. However, it depends largely on the performing skills of the operator. Moreover, many cancer foci in the prostate gland has very small size less than $1 \mathrm{~mm}^{9}$, whilst the spatial scales of current elastography techniques limit their applications to identify such small lesions. Alternatively, optical imaging can break the limit of ultrasound and magnetic resonance imaging (MRI) to achieve micro-scale resolution ${ }^{10}$.

Optical coherence tomography (OCT) is an emerging optical imaging technique which works similar to ultrasound except that OCT uses near-infrared light rather than acoustical waves. OCT enables micro-scale spatial resolution and millimetrescale imaging depth ${ }^{10}$. In the landmark paper published in 1998, Schmitt et al. ${ }^{11}$ first employed optical coherence tomography (OCT) ${ }^{12}$ to measure local displacement induced by quasi-static compression. Elastography with OCT is termed as optical coherence elastography (OCE). The early OCE studies relied on the pioneer work of Schmitt et al. using the speckle-tracking method with cross correlation technique to detect the tissue response after loading. Speckle is the finescale, random, mottled intensity pattern generated by the sub-resolution sample scatters present in all coherent imaging systems. Instead of using changes in the intensity information, phase-sensitive method obtains the phase information from complex OCT signal ${ }^{13}$. Phase-sensitive method has large displacement dynamic range, about 20 -fold greater than for the latter one ${ }^{14}$, as well as a high spatial resolution that is comparable to the underlying OCT system. Guan et al. ${ }^{15}$ combined phase-sensitive OCT with an ultrasound transducer to provide quantitative Young's modulus of a degenerated tendon model with a high spatial resolution. A high signal to noise ratio was reported to monitor the elasticity alteration of the tendon treated with collagenase of different concentration and time. It presented the clinical potential of using the method of the phase-sensitive OCE combined with vibration stimulation for disease evaluation.

Similarly, a preliminary work ${ }^{16}$ was conducted in ex vivo PCa detection for the first time with 120 biopsies from 10 patients by following the method of Guan et al. It proved that OCE can reliably differentiate benign and malignant prostate tissues with a high diagnostic accuracy. Additionally, the preliminary results demonstrated the distinct structural image and elastogram of biopsies of different Gleason score. Due to a small number of patients recruited, the previous work did not clearly show the impact of quantitative tissue stiffness on the diagnosis of Gleason score. In this paper, we present a large clinical study of 60 patients suspected with PCa using vibration OCE method. Building on the previous study, we will perform point by point comparison between 3D OCE images and histological images of each biopsy core. The quantitative Young's modulus value of the prostate biopsies will be statistically analysed based on the cancer aggressiveness. The aim of this continuing study aims to develop a quantitative biomarker to help the diagnosis of PCa more accurate.

\section{METHODOLOGY}

\section{Patients and biopsies}

We recruited 60 patients (age range 56-85) diagnosed with TRUS guided 12-core biopsy between April 2014 and December 2015, 11 of whom underwent RP afterwards at Ninewells Hospital. Ethical approval was granted by Tayside ethical committee (14/ES/0049). Informed consent was obtained from all the patients before their biopsy procedures for the reported study. According to the histopathologic report after the vibration OCE, there are 37 PCa patients, 1 patient with carcinosarcoma, and 22 patients not suspicious of malignancy whose biopsies were classified to the region of benign/normal biopsy tissues. Two combined Gleason scores are usually assigned to the PCa patients to evaluate the aggressiveness and prognosis of the cancer with a higher number to be more malignant. Wherein the lowest score given is 
Gleason $3+3=6{ }^{17}$ in the current clinical care. At least one non-cancerous prostate diseases were reported in 17 patients among the 22 cancer-free patients, for instance, benign prostatic hyperplasia (BPH), atypical small acinar proliferation (ASAP), prostatitis and/or prostatic intraepithelial neoplasia (PIN). Table 2 displays the details of patient number and percentage for each classification.

Table 1. Histopathologic classification of patients suspected with prostate cancer.

\begin{tabular}{|c|c|c|}
\hline Category & Number of patients & Percentage \\
\hline Prostate adenocarcinoma & 37 & $61.7 \%$ \\
\hline Carcinosarcoma & 1 & $1.7 \%$ \\
\hline Non-cancerous disease & 17 & $28.3 \%$ \\
\hline Disease-free & 5 & $8.3 \%$ \\
\hline Total & 60 & $100 \%$ \\
\hline
\end{tabular}

The 60 participants received standard 12-core based biopsies guided by TRUS from different regions of prostate gland. Overall, a total of 720 cores of tissue specimens were included in this study. The biopsy size was mainly determined by the geometry of the 18-gauge biopsy needle and the force applied during the operation procedure. Each biopsy was a circular core approximately 0.8-1.2 mm in diameter and 5-20 mm in length. For each patient, the 12 specimens were put into $10 \%$ neutral buffered formalin (50 $\mathrm{mL}$ ) immediately after TRUS procedure and stored in the independent containers. According to the previous study ${ }^{18}$ of the effect of fixative on tissue stiffness, the samples were embedded in the formalin for 24 hours before the elasticity test (OCE) was performed on each biopsy.

After the OCE imaging, biopsies were then sent to the pathology department at Ninewells Hospital preparing for the pathological analysis using a routine histological protocol. The haematoxylin and eosin (H\&E) staining was applied to exam the cellular structure and report the degree of malignancy. After processing the tissue, the $\sim 5 \mu \mathrm{m}$ section was mounted between a glass slide and coverslip. At least three histological slices were obtained from each biopsy. Lastly, the staging and percentage of cancer involvement were reported by an experienced pathologist (SL) blinded to the OCE data. A total 720 cores were categorised into two major groups according to the pathological results: namely non-cancerous prostate tissue (448 core biopsies) and malignant prostate tissue (272 core biopsies). Among them, there were 260 PCa samples which were further divided into 7 sub-groups as shown in Table 2. This sampling size with a varied spectrum should be sufficient for evaluating OCE imaging in the diagnosis of PCa with different aggressiveness.

Table 2. Histopathologic classification of biopsies from patients diagnosed with prostate cancer.

\begin{tabular}{|c|c|c|c|c|c|c|c|c|}
\hline Gleason score & $\mathbf{3 + 3}$ & $\mathbf{3 + 4}$ & $\mathbf{3 + 5}$ & $\mathbf{4 + 3}$ & $\mathbf{4 + 4}$ & $\mathbf{4 + 5}$ & $\mathbf{5 + 4}$ & Total \\
\hline Number of biopsies & 58 & 54 & 4 & 46 & 48 & 39 & 11 & 260 \\
\hline Percentage & $22.3 \%$ & $20.8 \%$ & $1.5 \%$ & $17.7 \%$ & $18.5 \%$ & $15.0 \%$ & $4.2 \%$ & $100 \%$ \\
\hline
\end{tabular}

\section{Optical Coherence Elastography (OCE)}

The OCE system consists of two main parts: signal detection and vibration stimulation as illustrated in Figure 1. For signal detection, a phase-sensitive optical coherence tomography (PhS-OCT) with spectral-domain configuration is adapted to measure the displacement of tissues. The PhS-OCT system employs a superluminescent diode (SLD, Thorlabs) as a broadband light source, with a centre wavelength of $\sim 1302 \mathrm{~nm}$ and bandwidth of $\sim 85 \mathrm{~nm}$. For the system performance, it provides an axial resolution of $8.8 \mu \mathrm{m}$ and a transverse resolution of $7.9 \mu \mathrm{m}$ in air. Additionally, the dynamic range of the PhS-OCT system is $\sim 100 \mathrm{~dB}$ at 0.5 -mm axial depth with a phase noise of 3 mrad. However, the signal to noise ratio (SNR) in the region of interest (ROI) of the tissue sample is $\sim 50 \mathrm{~dB}$. The acquisition rate is determined by the spectrometer of a maximum rate of $\sim 76,335 \mathrm{~A}$-scans/s. To generate vibration, a sine-wave signal modulated at $\sim 8 \mathrm{kHz}$ is generated by a function waveform generator (Agilent Technologies, USA) and an amplifier (AE Techron). The signal is then sent to drive a magnet shaker (Brüel \& Kjær Sound \& Vibration Measurement A/S, Denmark). The vibration is transmitted from the shaker to compress the sample and trigger vibration within the sample in the axial direction. 


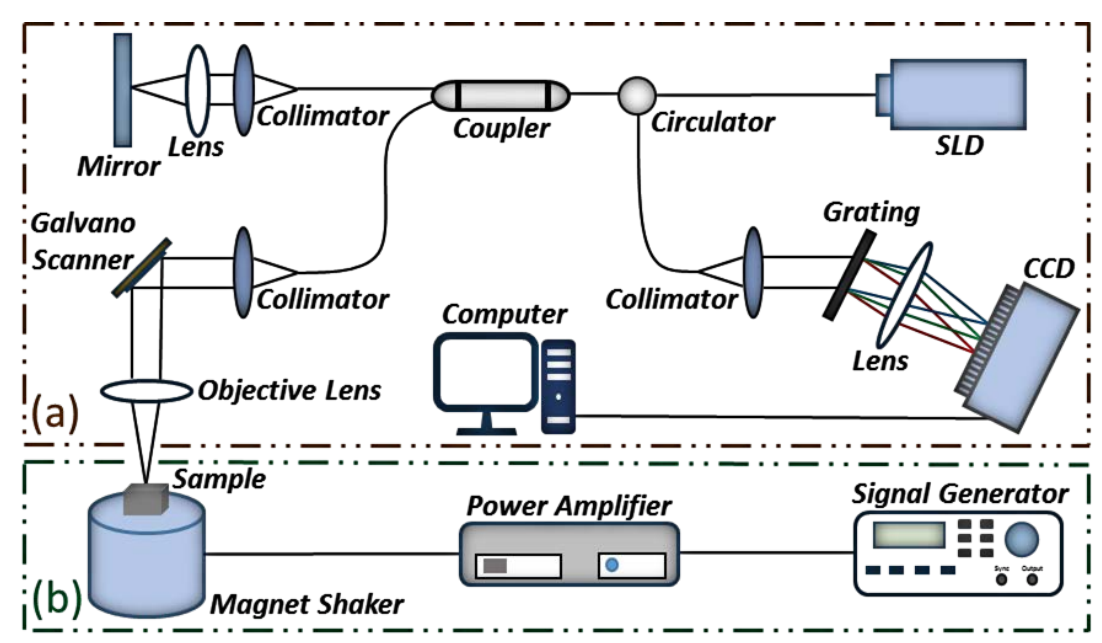

Figure 1. A schematic of system setup of the vibration OCE system consisting of (a) signal detection using PhS-OCT and (b) vibration stimulation using a shaker. SLD (superluminescent diode), CCD (charged coupled device) line-scan camera.

A schematic of the scanning protocol is shown in Figure 2 for the acquisition of a cross-sectional two-dimensional (2D) structure and elastogram. While the shaker continued to fire the stimulus to the sample, the vibration signal was acquired using M-B mode. To form an M-scan image, the PhS-OCT beam stayed at one location of the sample for 256 repeats. A complete B-scan (a cross-section view) consists of $256 \times 256$ A-scans. Three-dimensional (3D) elastogram was then realized with consecutive $B$ scans along the length of the prostate biopsy at an interval of $50 \mu$. Herein the acquisition time for a set of OCE data was 3 minutes for a size of $2 \mathrm{~mm} \times 2 \mathrm{~mm} \times 3 \mathrm{~mm}$. After the acquisition of one data set, the transitional stage was moved $2.5 \mathrm{~mm}$ in y direction for next acquisition, and repeated until the whole biopsy core was scanned. During the OCE scanning, the biopsy core was placed on $2 \%$ agar phantom with a thickness of $\sim 8 \mathrm{~mm}$ as an elasticity reference ${ }^{19}$. The total scanning time for each biopsy was dependent on the biopsy length, approximately 20 minutes on average. Finally, the raw structure and elastogram data sets were processed by MATLAB R2015b (The MathWorks, Natick, MA, USA) ${ }^{15}$ to generate structural and elastogram frames for each B-scan. The frames were then imported into Amira (Mercury Computer Systems, Berlin, Germany) and reconstructed into 3D data sets at full resolution.

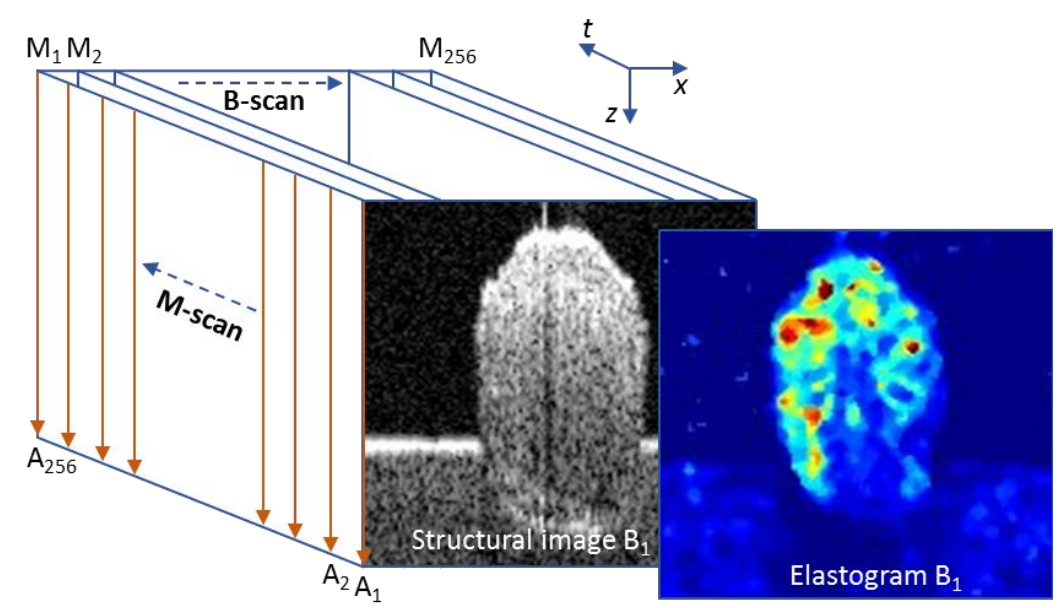

Figure 2. A schematic of the scanning protocol using M-B mode for the prostate biopsy tissue, wherein the agar phantom is used as a reference, $\mathrm{x}$-axis is the lateral position of the sample, $\mathrm{z}$-axis is the direction of laser beam, and t-axis represents the time location during M-scan. 
A systematic statistical method was conducted blinded to OCE and histological data to examine the reliability of quantitative OCE in differentiating benign and malignant prostate tissues as well as determining sensitivity and specificity of this technology. The estimated Young's modulus $(\mathrm{kPa})$ was compared among malignant and benign tissues, as well as different Gleason scores. All the analyses were performed with SPSS 22 (SPSS, Chicago, IL, USA). Differences between the groups were assessed using the Games-Howell test. $\mathrm{P}$ value of 0.05 was considered to be statistically significant.

\section{RESULTS}

\section{D elastogram}

The cancer involvement was reported by the histopathologist and the histological images were obtained by imaging the biopsy slide under the microscope with a fitted camera. The elastogram of a whole biopsy obtained from phase-sensitive OCE are compared with the histologic images in this study as demonstrated in Figure 3-7. Herein the Young's modulus of the biopsy is colour coded with red to be $\sim 300 \mathrm{kPa}$ and yellow to be $1000 \mathrm{kPa}$. In Figure 3, a benign biopsy is the control group for the other 4 malignant PCa biopsies of different Gleason score with a sum from 6 to 9 . The average stiffness of a benign biopsy is approximately $492.0 \mathrm{kPa}(\mathrm{SD}=44.4 \mathrm{kPa})$ as shown in Figure 3 indicated in dark red colour. In Figure 47 , the origin and end of the malignant area is labelled with the red arrows. As shown in the 4 biopsies, the malignant area in the 3D elastograms matches with that in the histologic images. Wherein, the colour enhancement of the elastogram from light yellow to bright yellow was observed in the malignant region of the PCa biopsies from Figure 4 to Figure 7 . It indicates an increase of Gleason sum and cancer involvement with the rise of Young's modulus.
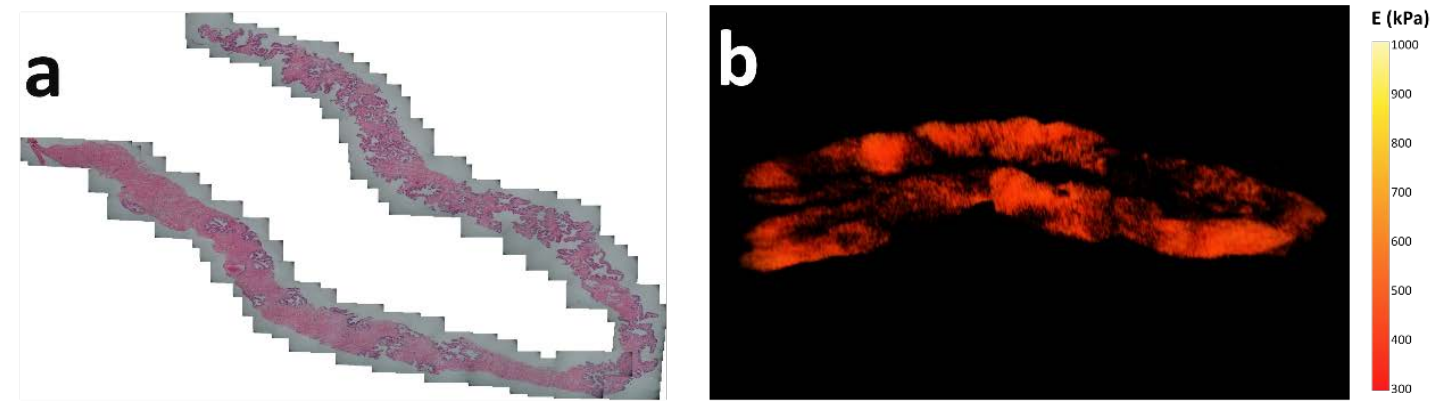

Figure 3. Comparison between histologic image (a) and 3D elastogram (b) of benign biopsy
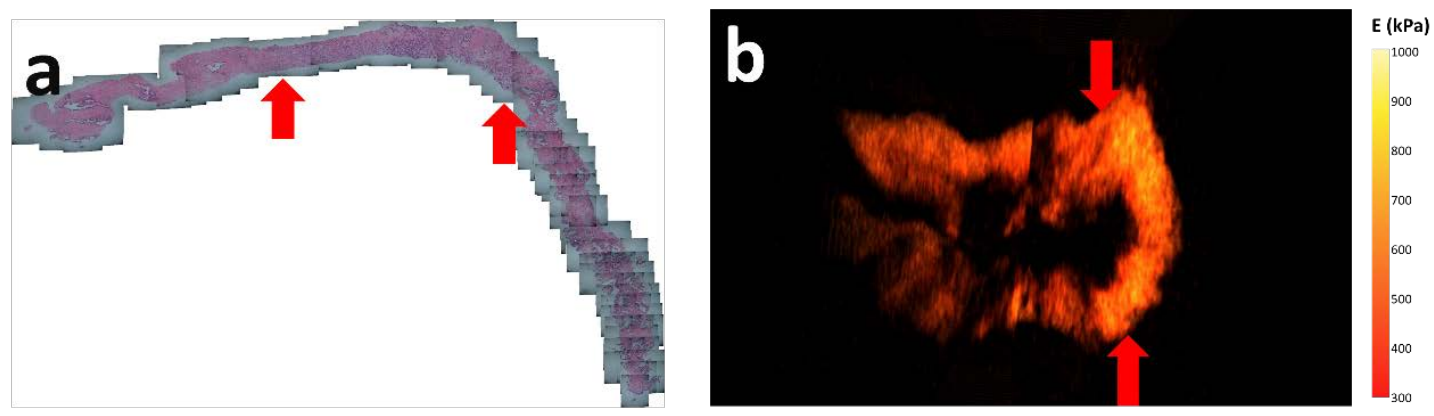

Figure 4. Comparison between histologic image (a) and 3D elastogram (b) of PCa biopsy of Gleason score 3+3 with 30\% cancer involvement. The red arrows indicate the start and end of malignancy. 

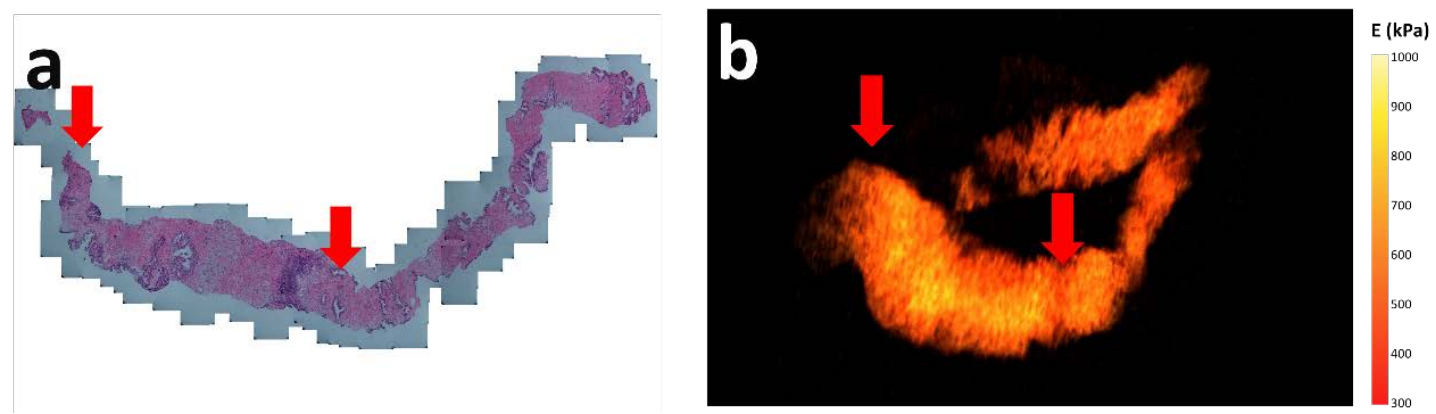

Figure 5. Comparison between histologic image (a) and 3D elastogram (b) of PCa biopsy of Gleason score 3+4 with $40 \%$ cancer involvement. The red arrows indicate the start and end of malignancy.
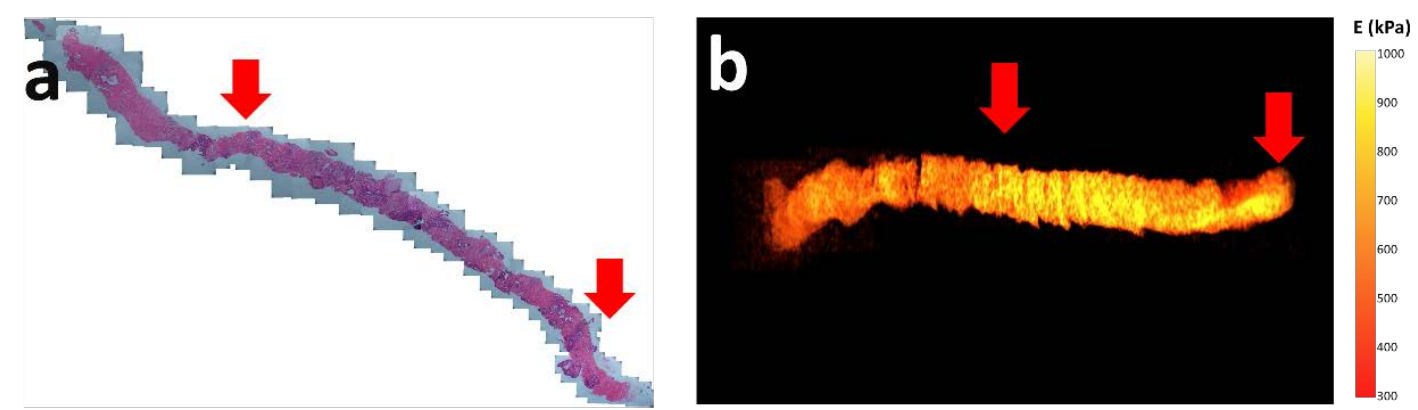

Figure 6. Comparison between histologic image (a) and 3D elastogram (b) of PCa biopsy of Gleason score $4+4$ with $60 \%$ cancer involvement. The red arrows indicate the start and end of malignancy.
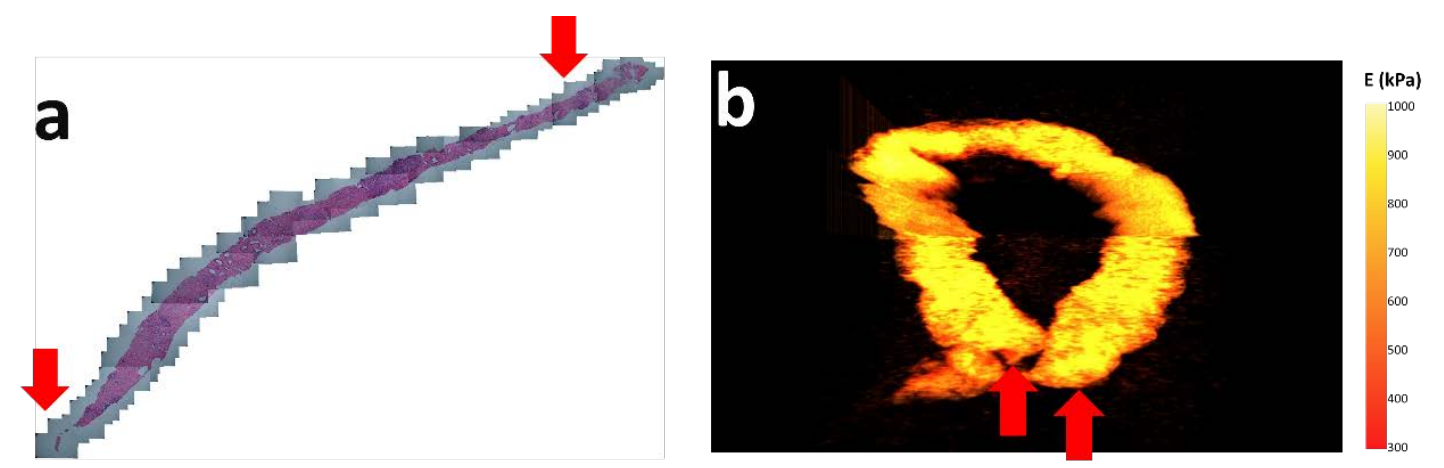

Figure 7. Comparison between histologic image (a) and 3D elastogram (b) of PCa biopsy of Gleason score $4+5$ with $80 \%$ cancer involvement. The red arrows indicate the start and end of malignancy.

\section{Statistical analysis}

To validate the diagnostic accuracy of the vibration OCE method, the weighted average Young's modulus value was calculated over the whole area within each biopsy. The average Young's modulus of cancer and benign biopsies is 698.43 $(\mathrm{SD}=125.29 \mathrm{kPa})$ and $443.07(\mathrm{SD}=88.95 \mathrm{kPa}$ ) respectively. Results from PCa and benign prostate tissue are illustrated with the whisker plot in Figure 8 (a). A significant increase of stiffness can be observed $(p<0.001)$ between benign and PCa tissue. In Figure 8 (a), PCa is suspected with the Young's modulus higher than $600 \mathrm{kPa}$ (cutoff) after comparing the elasticity data obtained from OCE and the corresponding histological report. Hence, the biopsies of the Young's modulus value higher than $600 \mathrm{kPa}$ are treated as positive result with $\mathrm{PCa}$, and the others are negative result of cancer-free biopsies. 
The data analysis shows that the sensitivity and specificity are $89.6 \%$ and $99.8 \%$ using $600 \mathrm{kPa}$ as a threshold. The relationship between Gleason score and the estimated Young's modulus is compared in Figure 8 (b). There is a significant difference noticed among Young's modulus of different Gleason scores estimated by OCE (p Value $<0.05$ ) except for that of Gleason score 7 and 8 (p value $=0.765$ ).
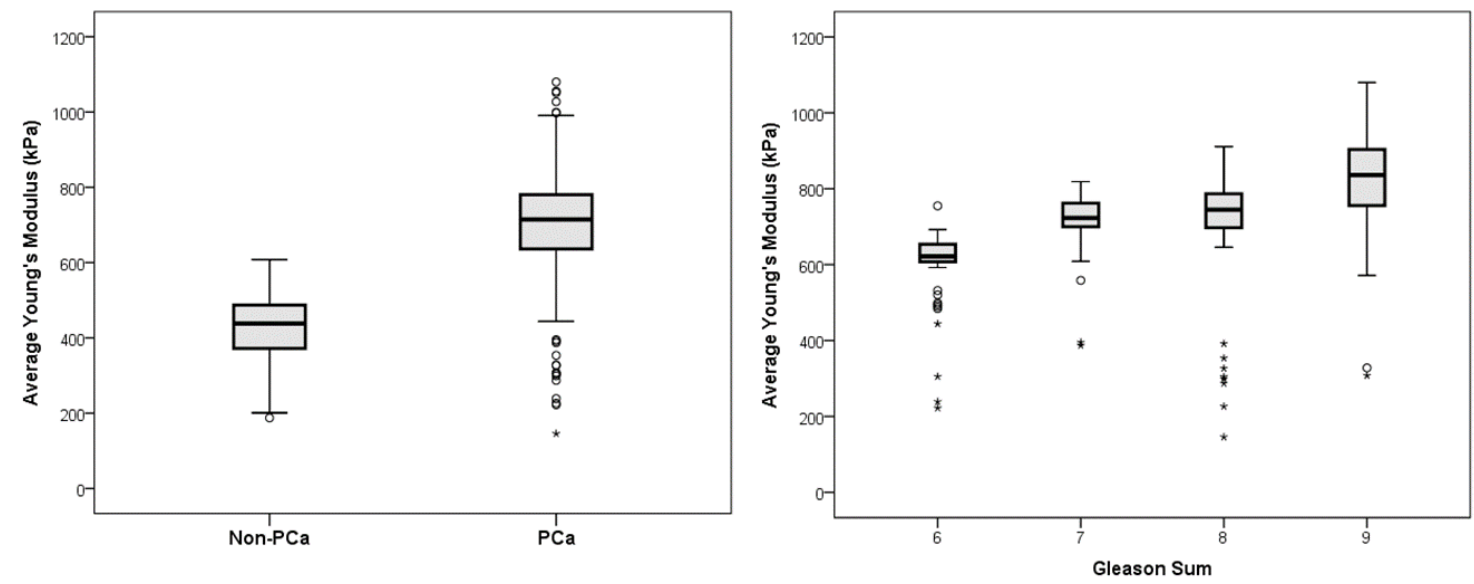

Figure 8. Young’s modulus comparison of (a) benign and malignant biopsies and (b) biopsies of different Gleason sum.

\section{DISCUSSION AND CONCLUSION}

This study utilized a novel optical imaging method, the phase-sensitive OCE with vibration, to estimate the mechanical properties of the prostate tissue. The estimated tissue stiffness was used to categorise specimens into different histological classifications. Quantitative 3D elastogram of a prostate biopsy can also be reconstructed in high resolution from consecutive 2D elastograms. Using a threshold of $600 \mathrm{kPa}$, the vibration OCE shows promising ability in PCa detection with a high sensitivity and specificity. Additionally, both the 3D elastograms and the statistical analysis illustrates an ascending trend of the quantitative stiffness with the increase of Gleeson scores.

As noted the prostate biopsies in this study are fixed in the formalin solution before the OCE evaluation, but the tissues should be fixed to the same extend after 24 hours according to our previous study ${ }^{18}$. The diagnostic accuracy analysed in this study is relied on the histopathological verification requiring the knowledge and experience of the histopathologist. However, the early cancer with the pattern of Gleason score smaller than 3 is difficult to be distinguished by naked eyes and as a result the lowest Gleason score reported is $3+3$ in the current clinical care. This can be the explanation of the case when the benign prostate biopsy has high stiffness detected by the vibration OCE in this study.

In conclusion, this study presents the vibration OCE to estimate the Young's modulus of prostate biopsies from 60 patients suspected with PCa. Based on a total of 720 biopsies, the results proved that this method is capable to differentiate benign from malignant biopsies with a high diagnostic accuracy, as well as quantify the cancer malignancy. Thus, the Young's modulus of the prostate biopsy is potential to be used as a mechanical biomarker to detect PCa and distinguish cancer with different aggressiveness. On the basis of the findings from this study, a future perspective is the in-vivo study with the OCE needle probe to realise real-time diagnosis of PCa and accurate characterisation of the malignancy.

\section{ACKNOWLEDGEMENTS}

We want to express our gratitude to the patients and clinicians who have contributed to this study. This work was supported by a Scottish Universities Physics Alliance (SUPA) PhD studentship and grants from Prostate Cancer UK (PCUK). 


\section{REFERENCES}

[1] F. May, T. Treumann, P. Dettmar et al., "Limited value of endorectal magnetic resonance imaging and transrectal ultrasonography in the staging of clinically localized prostate cancer,” Bju International, 87(1), 6669 (2001).

[2] M. S. Cohen, R. S. Hanley, T. Kurteva et al., "Comparing the Gleason prostate biopsy and Gleason prostatectomy grading system: the Lahey Clinic Medical Center experience and an international meta-analysis," Eur Urol, 54(2), 371-81 (2008).

[3] E. C. Serefoglu, S. Altinova, N. S. Ugras et al., "How reliable is 12-core prostate biopsy procedure in the detection of prostate cancer?,” Can Urol Assoc J, 7(5-6), E293-8 (2013).

[4] P. A. Humphrey, "Gleason grading and prognostic factors in carcinoma of the prostate," Modern Pathology, 17(3), 292-306 (2004).

[5] T. A. Krouskop, T. M. Wheeler, F. Kallel et al., "Elastic moduli of breast and prostate tissues under compression,” Ultrason Imaging, 20(4), 260-74 (1998).

[6] T. Shiina, K. R. Nightingale, M. L. Palmeri et al., "WFUMB Guidelines and Recommendations for Clinical Use of Ultrasound Elastography: Part 1: Basic Principles and Terminology,” Ultrasound in Medicine \& Biology, 41(5), 1126-1147 (2015).

[7] J. Ophir, I. Cespedes, H. Ponnekanti et al., "Elastography: a quantitative method for imaging the elasticity of biological tissues," Ultrason Imaging, 13(2), 111-34 (1991).

[8] T. Varghese, "Quasi-Static Ultrasound Elastography,” Ultrasound Clin, 4(3), 323-338 (2009).

[9] A. Sarvazyan, T. J. Hall, M. W. Urban et al., "An Overview of Elastography - an Emerging Branch of Medical Imaging,” Curr Med Imaging Rev, 7(4), 255-282 (2011).

[10] B. F. Kennedy, K. M. Kennedy, and D. D. Sampson, "A Review of Optical Coherence Elastography: Fundamentals, Techniques and Prospects," Selected Topics in Quantum Electronics, IEEE Journal of, 20(2), 272-288 (2014).

[11] J. Schmitt, “OCT elastography: imaging microscopic deformation and strain of tissue,” Opt Express, 3(6), 199211 (1998).

[12] J. G. Fujimoto, M. E. Brezinski, G. J. Tearney et al., "Optical biopsy and imaging using optical coherence tomography,” Nat Med, 1(9), 970-2 (1995).

[13] Y. H. Zhao, Z. P. Chen, C. Saxer et al., "Phase-resolved optical coherence tomography and optical Doppler tomography for imaging blood flow in human skin with fast scanning speed and high velocity sensitivity," Optics Letters, 25(2), 114-116 (2000).

[14] B. F. Kennedy, S. H. Koh, R. A. McLaughlin et al., "Strain estimation in phase-sensitive optical coherence elastography,” Biomedical Optics Express, 3(8), 1865-1879 (2012).

[15] G. Guan, C. Li, Y. Ling et al., "Quantitative evaluation of degenerated tendon model using combined optical coherence elastography and acoustic radiation force method,” J Biomed Opt, 18(11), 111417 (2013).

[16] C. Li, G. Guan, Y. Ling et al., "Detection and characterisation of biopsy tissue using quantitative optical coherence elastography (OCE) in men with suspected prostate cancer," Cancer Lett, 357(1), 121-8 (2015).

[17] J. I. Epstein, L. Egevad, M. B. Amin et al., "The 2014 International Society of Urological Pathology (ISUP) Consensus Conference on Gleason Grading of Prostatic Carcinoma Definition of Grading Patterns and Proposal for a New Grading System,” American Journal of Surgical Pathology, 40(2), 244-252 (2016).

[18] Y. Ling, C. Li, K. Feng et al., "Effects of fixation and preservation on tissue elastic properties measured by quantitative optical coherence elastography (OCE),” J Biomech, 49(7), 1009-1015 (2016).

[19] C. Li, G. Guan, R. Reif et al., "Determining elastic properties of skin by measuring surface waves from an impulse mechanical stimulus using phase-sensitive optical coherence tomography,” J R Soc Interface, 9(70), 831-41 (2012). 